\title{
Cardiac conduction system anomalies and sudden cardiac death: insights from murine models
}

\section{Amelia Aránega *, Angel J. De La Rosa and Diego Franco}

Department of Experimental Biology, Faculty of Experimental Sciences, University of Jaén, Jaén, Spain

Edited by:

Carol Ann Remme, University of

Amsterdam, Netherlands

\section{Reviewed by:}

Carmen Valenzuela, Instituto de

Investigaciones Biomédicas

CSIC-UAM, Spain

Marina Cerrone, NYU School of

Medicine, USA

*Correspondence:

Amelia Aránega, Department of

Experimental Biology, Faculty of

Experimental Sciences, University of

Jaén, Paraje de las Lagunillas s/n,

23071 Jaén, Spain.

e-mail: aaranega@ujaen.es
The cardiac conduction system (CCS) is composed of a group of myocardial tissues that control and coordinate the heart. Alterations in the CCS - especially in the His-Purkinje system, have been identified as a major cause of lethal arrhythmias. Unstable arrhythmias secondary to channelopathies significantly increase the risk of sudden cardiac death (SCD). SCD is a major contributor to mortality in industrialized countries, and most cases of SCD in the young are related to inherited ion channel diseases. In this paper, we review a series of studies with murine transgenic models that revealed that some arrhythmias are associated with the CCS and may lead to SCD

Keywords: cardiac conduction system, ion channel, sudden death

\section{INTRODUCTION}

Sudden cardiac death (SCD) occurs when an apparently healthy person unexpected dies, which occurs within a very short time interval - usually $1 \mathrm{~h}$ after symptom onset. Although sudden death in infants, children, and young adults is relatively rare with an incidence of one to five cases per 100,000 population per year, up to 7,000 asymptomatic children die every year in the USA. In almost half of the cases no warning signs or symptoms were detected. Although structural cardiovascular abnormalities explain most cases of SCD in young people, the cause of death remains unexplained after autopsy in 10-30\% of cases (Kauferstein et al., 2009). Cases of unexplained SCD in young people are mostly due to inherited ion channel diseases such as long QT syndrome (LQTS), short QT syndrome (SQTS), Brugada syndrome, and catecholaminergic polymorphic ventricular tachycardia (CPVT) (Krous et al., 2004; Basso et al., 2009).

The cardiac conduction system (CCS) generates and conducts electrical signals (action potentials) throughout the heart to trigger and coordinate the heartbeat. The electrical activity of the heart behaves like the electrical activity of any other biological tissue, and is ultimately dependent on the expression of ion channels in the myocytes forming the CCS. Ion channel expression defines the characteristic action potentials generated by these specialized myocytes, and is clearly different from that in the working myocardium (Boyett, 2009). Interestingly, several recent reports have pointed out that ion channel expression remodeling within the different CCS components may be responsible for the bradycardia and the increase in atrioventricular block with aging (Muir et al., 2005, Abd Allah et al., 2007). There is growing evidence that the different components of the CCS (e.g., Purkinje fibers network) play a pivotal role in both the initiation and perpetuation of several arrhythmias such as ventricular fibrillation (VF; Nogami, 2001). In addition, recent mapping studies have shown that Purkinje fibers can initiate VF in some patients with pro-arrhythmogenic syndromes: Brugada syndrome, LQTS (Haïssaguerre et al., 2003). Despite the significant role of ion channels in the electrical activity of CCS, their influence and contribution to CCS dysfunctions are still poorly understood.

\section{ELECTRICAL CONFIGURATION OF THE CARDIAC CONDUCTION SYSTEM}

The heart achieves coordinated contraction of atrial and ventricular chambers thanks to the precise timing of firing of the CCS; it is a specialized complex and heterogeneous network of cells that initiate and allow propagation of action potentials through the heart (Gourdie et al., 2003). The normal cardiac impulse in vertebrates' heart originates in the pacemaker cells of the sinoatrial node (SAN) located in the right atrium (Anderson et al., 1983). The impulse is then conducted through the atrium to the atrioventricular junction, where impulses are delayed through specialized slow conducting cardiomyocytes in the atrioventricular node (AVN). This delay is necessary to allow time for the atrial chambers to fully contract and pump blood across $\mathrm{AV}$ valves before the occurrence of ventricular contraction (Hatcher and Basson, 2009). After AVN delay, the electrical signal is propagated to the ventricles along bundles of specialized conduction tissue to the distal Purkinje fibers, which ramify among the contractile myocardium. The tips of the Purkinje fibers are electrically coupled to muscle cells and the working myocytes are longitudinally connected via gap junctions, thereby initiating a coordinated contraction of the ventricles (Pennisi et al., 2002).

It is interesting to remark that the SA node is a complex and irregular structure. There is regional heterogeneity in the SAN in mammals in terms of cell morphology, pacemaker activity, action potential configuration and conduction, ionic current densities (INa ICa, L, Ito, Iks, If), gap junction protein expression (Cx40, 
Cx43, Cx45), and autonomic regulation (Hall et al., 2004; Herfst et al., 2004). Moreover, although this bundle is located in the AV junction area, its molecular phenotype shows most of the characteristics of the working ventricular myocardium, displaying a high conduction velocity compared to the slower conducting AV nodal tissue (Franco and Icardo, 2001).

For instance, in ventricular and atrial myocytes and Purkinje fibers, the upstroke of the action potential (phase 0 ) is rapid and results from the activation of voltage-gated $\mathrm{Na}^{+}$(Nav) channels. However, phase 0 is markedly slower in the pacemaker cells of the SAN and AVN suggesting that Nav channels do not play a relevant role in depolarization (Baruscotti et al., 2005). These findings are consistent with the heterogeneity found in $\mathrm{Na}^{+}$channel expression within the CCS (Domínguez et al., 2005, 2008).

Phase 0 of the action potential in Purkinje fibers and in atrial and ventricular myocytes is followed by a transient repolarization (phase 1), reflecting Nav channel inactivation and the activation of the fast transient voltage-gated outward $\mathrm{K}^{+}$current (Ito, $\mathrm{f}$ ). This transient repolarization or "notch," which can be quite prominent in Purkinje and ventricular cells, influences the height and duration of the action potential plateau (phase 2). Membrane depolarization also activates voltage-gated $\mathrm{Ca}^{2+}$ (Cav) currents; $\mathrm{Ca}^{2+}$ influx through L-type Cav channels during the phase 2 plateau is the main trigger for excitation-contraction coupling in the working myocardium. Thus, $\mathrm{Ca}^{2+}$ entering through L-type Cav triggers a relatively larger release of $\mathrm{Ca}^{2+}$ from the sarcoplasmic reticulum (SR) via intracellular $\mathrm{Ca}^{2+}$ release channels (ryanodine receptors, RyRs). The increase in $\left[\mathrm{Ca}^{2+}\right] \mathrm{i}$ promotes cell contraction by $\mathrm{Ca}^{2+}$ binding to contractile proteins (Moosmang et al., 2001). In SAN and AVN cells, activation of (L-type) Cav channels also contributes to action potential generation, particularly in cells expressing low levels of functional Nav channels (Moosmang et al., 2001).

The driving force for $\mathrm{K}^{+}$efflux is high during the plateau phase of the action potential in the ventricular and atrial myocardium; when Cav channels become inactive, outward $\mathrm{K}^{+}$currents predominate resulting in repolarization (phase 3 ), and bringing membrane voltage back to the resting potential. In contrast to Nav and Cav currents, there are multiple types of voltage-gated $\mathrm{K}^{+}$ $(\mathrm{Kv})$ currents, as well as non-voltage-gated, inwardly rectifying $\mathrm{K}^{+}$(Kir) currents that contribute to myocardial action potential repolarization: the greatest functional diversity is among $\mathrm{Kv}$ channels.

At least two types of transient outward currents - Ito, $\mathrm{f}$ and Ito, s - and several components of delayed rectification - including IKr [IK(rapid)], IKs [IK(slow)], and IKur [IK(ultrarapid), among others] - have been identified. However the timing and voltage-dependent properties of the different $\mathrm{Kv}$ currents identified in different regions of the heart are remarkably similar. This suggests that the same (or very similar) molecular entities contribute to the generation of each of the $\mathrm{Kv}$ channels in different cells. Relative $\mathrm{Kv}$ channel expression levels in cardiac cells vary according to the region of the heart (i.e., atria, ventricles); this heterogeneity significantly contributes to the observed regional differences in action potential waveforms (Stieber et al., 2003; Aiba and Tomaselli, 2010).

\section{CARDIAC CONDUCTION SYSTEM, ARRHYTHMOGENESIS, AND SUDDEN CARDIAC DEATH: CONTRIBUTIONS FROM MURINE MODELS}

In the CCS, ion channels are specialized and differ from those in the working myocardium, as they provide the electrical activity required for its proper function. Although changes in the expression and/or configuration of ion channels and connections within the conduction system may have fatal consequences - they can generate arrhythmogenic processes - the role of ion channels in CCS dysfunction remodeling is not yet well understood. For instance, it is noteworthy that in an aging heart, there are deleterious changes in CCS remodeling that are a contributing factor to the relatively high prevalence of cardiac arrhythmias, which increases the risk of SCD in the aging population (Dobrzynski et al., 2007). Furthermore, it is well known that athletes have a lower heart rate than non-athletes, and sinoatrial node remodeling in athletes can become pathological and result in a sick sinus syndrome (Baldesberger et al., 2008). Although there is growing evidence supporting that molecular remodeling of electro-generating, propagating, and coupling channels, as well as CCS structural remodeling may be behind cardiac arrhythmias (Thomas et al., 2007), the mechanism by which CCS remodeling takes place is still unknown.

With the development of transgenic animal technology, a growing number of experimental mouse/rat models have emerged. However, as the cardiac electrophysiological characteristics of mice are significantly different from those of humans, these models have substantial limitations - e.g., the duration of cardiac action potential is very short in mice; there is no plateau phase, and the heart rate is extremely high (resting heart rate: $600 \mathrm{bpm}$ ) in mice. Despite these differences, over the past 15 years, genetically engineered mouse models have been developed to elucidate the role of different ion channels in arrhythmogenic pathologies. The results obtained suggest that CCS components play a major role in the generation of cardiac arrhythmias. In this paper, we describe the main mouse models with ion channel alterations in which a CCS dysfunction leads to an arrhythmogenic substrate (Table 1).

\section{MOUSE MODELS OF ION CHANNELS REMODELING LINKED TO NODE TISSUE DYSFUNCTION}

A number of mouse models have been developed to elucidate the role of different ion channels in node tissue and in the generation of arrhythmias. For instance, different mouse transgenic models have been developed with the aim of identifying the relationship between Hcn channel alterations and pacemaker activity. The homozygous embryo mice lacking the exon 4 of the Hcn 4 gene - which encodes ion channel pore and transmembrane segment six - died in the uterus, thus indicating that Hcn4 is essential for the normal development of the heart (Stieber et al., 2003). In contrast, the role of If in adult mice is controversial. As Hcn4 knockout mice fail to develop a normal heart, it has not been until recently that we have elucidated whether If has the same prominent role in adult as in embryonic mice.

Accordingly, Herrmann et al. (2007) developed an adult mouse lacking Hcn 4 channels in a temporally controlled manner. Deletion of Hcn4 in adult mice eliminated most of sinoatrial If and resulted in a cardiac arrhythmia characterized by recurrent sinus pauses. However, these mutants showed no impairment in heart 
Table 1 | Mouse transgenic models with ion channel deficiency and CCS dysfunction.

\begin{tabular}{|c|c|c|}
\hline Mouse model & Ion channel deficiency/location & Arrhythmogenic phenotype \\
\hline Hcn4-/- mice (Stieber et al., 2003) & Hcn4/systemic & Died in utero \\
\hline Hcn4-inducible-knockout mice (Herrmann et al., 2007) & Hcn4/whole heart & Recurrent sinus pauses \\
\hline Hcn4-KiT mice (Hoesl et al., 2008) & $\mathrm{Hcn} 4 / \mathrm{CCS}$ & Normal cardiac rhythm \\
\hline ciHcn4-KO (Baruscotti et al., 2011) & Hcn4/whole heart & Severe bradycardia and AV block \\
\hline Scn5a+/- mice (Hao et al., 2011; Martin et al., 2011) & Nav1.5/systemic & $\begin{array}{l}\text { Reduced sinoatrial node automaticity/polymorphic } \\
\text { ventricular tachycardia }\end{array}$ \\
\hline R176Q/+ knock-in mice (Mathur et al., 2009) & Ryr2 mutation/systemic & Sudden cardiac death \\
\hline RyR(R4496C) mice (Cerrone et al., 2005, 2007) & Ryr2 mutation/systemic & CPVT/DAD activity in Purkinje fibers \\
\hline
\end{tabular}

rate acceleration during sympathetic stimulation, thus revealing that, in adult mice, Hcn 4 does not appear to be the only mechanism involved in the adrenergic stimulation of sinus activity.

Moreover, conduction system-specific deletion of Hcn4 channels using the KiT transgene did not result in bradycardia (Hoesl et al., 2008). This supports the notion that Hcn4 channels mainly provide the depolarizing currents necessary to counterbalance an increase in repolarizing currents in the CCS. On the contrary, in an inducible and cardiac-specific Hon 4 knockout (ciHcn4-KO) mouse model, ablation of $\mathrm{Hcn} 4$ consistently led to the progressive development of severe bradycardia ( $\sim 50 \%$ reduction of original rate) and AV block, eventually leading to heart arrest and death in about 5 days. This outcome suggests that cardiac Hcn 4 channels are essential for normal heart impulse generation and conduction in adult mice; in addition, these results support the notion that dysfunctional Hcn 4 channels can be a direct cause of rhythm disorders, which lead to sudden death events (Baruscotti et al., 2011). These contradictory results might be partially due to the nature of the mouse models used in these studies, as they were designed to lack a Hcn channel within the whole heart, and/or within both the SAN and AVN (Table 1). The development of Cre deleter mouse lines - which can render sinoatrial-specific Hcn channel deletion - will help to better understand the role of Hcn channels in pacemaker activity.

Knockout mouse models for other key cardiac ion channels are also useful tools for evaluating how a misexpression of specific ion channels can generate an arrhythmogenic substrate. Thus, Nav1.5 deficiency in heterozygous Scn5a knockout [Scn5a $(+/-)]$ mice resulted in reduced SAN automaticity, which suggests that the loss of expression/function of sodium currents can severely impair action potentials in pacemaker cells. Moreover, downregulation of a wide range of other ion channels and related transcripts in $\operatorname{Scn} 5 \mathrm{a}(+/-)$ mice suggest that impaired Scn5a expression may lead to significant electric remodeling in the sinus node (Hao et al., 2011).

In addition, the contribution of transcription factors to proper CCS embryonic development is being widely explored. Previous studies have described that the T-box transcription factor Tbx3 is predominantly expressed in developing and mature pacemakers [SAN and AV canal (AVC) and node], as well as in the AVB and branches. Tbx3 is sufficient to induce the formation of ectopic pacemakers in the atrial myocardium during its development (Hoogaars et al., 2004, 2007). These data suggest a role for Tbx3 in mammalian cardiac development and CCS function. More recently, the generation of a unique series of Tbx3 hypomorphic and conditional mouse mutants (Frank et al., 2011) revealed that the development of the CCS structure and function is extremely sensitive to Tbx3, to the extent that a decrease in Tbx3 below a critical level causes lethal embryonic arrhythmias; these arrhythmias are accompanied by disrupted expression of multiple ion channels such as voltage-gated sodium channels ( $\mathrm{Scn} 7 \mathrm{a})$ and Connexin30.2. Furthermore, surviving Tbx 3 mutants are at an increased risk for sudden death, thus suggesting that TBX3 may be a candidate gene for human arrhythmia syndromes.

\section{MOUSE MODELS OF ION CHANNEL REMODELING LINKED TO PURKINJE SYSTEM DYSFUNCTION}

Catecholaminergic polymorphic ventricular tachycardia is an inherited disease leading to arrhythmias and SCD. The autosomal dominant form has been associated with ryanodine receptor gene (RyR2) mutations, leading to increased spontaneous $\mathrm{Ca}^{2+}$ release from the SR (Priori et al., 2001). Mice heterozygous for the R4496C mutation (RyR2/RyR2R4496C) recapitulate the human phenotype of CPVT by developing ventricular tachycardia and/or VF under adrenergic stimulation (Cerrone et al., 2005). More recently, epicardial and endocardial optical mapping analyses have demonstrated that arrhythmias in RyR2/RyR2R4496C mice are mediated by delayed afterdepolarization (DAD)-induced triggered activity occurring in Purkinje fibers; this reveals that the His-Purkinje system is an important source of focal arrhythmias in CPVT (Cerrone et al., 2007). Some authors have suggested that RYR2 mutations may cause "arrhythmogenic right ventricular dysplasia type 2" (ARVD2), a condition characterized by fibro-fatty degeneration of the right ventricle and by the presence of ventricular arrhythmias. The generation of a knock-in mouse model with the R176Q mutation (a human Ryr2 mutation previously identified in a 15-year-old male with ARVD2) led us to better understand the relationship between Ryr2 mutations and ARVD2. During the experiment, R176Q/+ knock-in mice displayed an increased incidence of sudden unexpected death during the first weeks of life, and electrophysiological recordings displayed cardiac arrhythmias in $\mathrm{R} 176 \mathrm{Q} /+$ mice associated with SCD (Mathur et al., 2009). In another study, Kang et al. (2010) crossed RyR2R4496C/+ CPVT mutant mice with Cntn2-EGFP BAC transgenic mice (which express a fluorescent reporter gene in cells of the CCS). Interestingly, Kang's study revealed that the RyR2(R4496C/+) Purkinje cells display a greater propensity to develop abnormalities in intracellular 
$\mathrm{Ca}^{2+}$ handling than ventricular myocytes. This proarrhythmic behavior was enhanced by disease-causing mutations in the RyR2 $\mathrm{Ca}^{2+}$ release channel and greatly exacerbated by catecholaminergic stimulation, with the development of arrhythmogenic triggered beats.

Finally, very recent experiments carried out using the $\operatorname{Scn} 5 a+/-$ mouse model - which has been shown to closely reproduce many of the key features of Brugada syndrome (a genetic channelopathy strongly linked to SCD) in humans - have revealed that Scn5a+/mice display lines of conduction block across the RV resulting from premature ventricular beats led to the formation of reentrant circuits and polymorphic ventricular tachycardia (Martin et al., 2011). Some authors have reported that the Purkinje system plays a major role in the development of VF in patients with LQTS and Brugada syndrome (Haïssaguerre et al., 2003). Consequently, the results obtained suggest that the Purkinje system might

\section{REFERENCES}

Abd Allah, E. S.H., Boyett, M. R., and Dobrzynski, H. (2007). Postnatal developmental changes in rabbit sinoatrial and atrioventricular nodes. J. Mol. Cell. Cardiol. 42, S2.

Aiba, T., and Tomaselli, G. F. (2010) Electrical remodeling in the failing heart. Curr. Opin. Cardiol. 25, 29-36.

Anderson, R. H., Ho, S. Y., and Becker, A. E. (1983). The surgical anatomy of the conduction tissues. Thorax 38, 408-420.

Baldesberger, S., Bauersfeld, U., Candinas, R., Seifert, B., Zuber, M., Ritter, M., Jenni, R., Oechslin, E., Luthi, P., Scharf, C., Marti, B., and Attenhofer Jost, C. H. (2008). Sinus node disease and arrhythmias in the long-term follow-up of former professional cyclists. Eur. Heart J. 29, 71-78.

Baruscotti, M., Bucchi, A., and DiFrancesco, D. (2005). Physiology and pharmacology of the cardiac pacemaker ("funny") current. Pharmacol. Ther. 107, 59-79.

Baruscotti, M., Bucchi, A., Viscomi, C., Mandelli, G., Consalez, G., GnecchiRusconi, T., Montano, N., Casali, K. R., Micheloni, S., Barbuti, A., and DiFrancesco, D. (2011). Deep bradycardia and heart block caused by inducible cardiac-specific knockout of the pacemaker channel gene Hcn4. Proc. Natl. Acad. Sci. 108, 1705-1710.

Basso, C., Carturan, E., Pilichou, K., Rizo, S., Corrado, M., and Thiene, G. (2009). Sudden cardiac death with normal heart: molecular autopsy. Cardiovasc. Pathol. 19, 321-325.

Boyett, M. R. (2009). "And the beat goes on." The cardiac conduction system: the wiring system of the heart. Exp. Physiol. 94, 1035-1049.
Cerrone, M., Colombi, B., Santoro, M., di Barletta, M. R., Scelsi, M., Villani, L., Napolitano, C., and Priori, S. G. (2005). Bidirectional ventricular tachycardia and fibrillation elicited in a knock-in mouse model diac ryanodine receptor. Circ. Res. 96, e77-e82.

Cerrone, M., Noujaim, S. F., Tolkacheva, E. G., Talkachou, A., O'Connell, R., Berenfeld, O., Anumonwo, J., Pandit, S. V., Vikstrom, K., Napolitano, C., Priori, S. G., and Jalife, J. (2007). Arrhythmogenic mechanisms in a mouse model of catecholaminergic polymorphic ventricular tachycardia. Circ. Res. 101, 1039-1048.

Dobrzynski, H., Boyett, M. R., and Anderson, R. H. (2007). New insights into pacemaker activity: promoting understanding of sick sinus syndrome. Circulation 115, 1921-1932.

Domínguez, J. N., Navarro, F., Franco, D., Thompson, R. P., Aránega, A. E. (2005). Temporal, and spatial expression pattern of SCN1b during heart development. Cardiovasc. Res. $65,842-850$.

Domínguez, J. N., Rosa, A. D., Navarro, F., Franco, D., Aránega, A. E. (2008). Tissue distribution, and subucelullar localization of the cardiac sodium channel during mouse heart development. Cardiovasc. Res. 78, 45-52.

Franco, D., and Icardo, J. M. (2001). Molecular characterization of the ventricular conduction system in the developing mouse heart: topographical correlation in normal and congenitally malformed hearts. Cardiovasc. Res. 49, 417-429.

Frank, D. U., Carter, K. L., Thomas, K. R., Burr, R. M., Bakker, M. carrier of a mutation in the car-

play a significant role in the outset of the electrophysiological abnormalities found in Scn5+/- mice.

\section{CONCLUDING REMARKS}

There is growing evidence that ion channel remodeling within the CCS may play a crucial role in the generation of cardiac arrhythmias associated with SCD. A number of mouse transgenic models have been developed to analyze the role of the different ion channels in the development of specific arrhythmias. Studies with these animal models have revealed that the arrhythmias associated with changes in ion channel expression/function within the heart are mainly caused by an impaired CCS component. Therefore, an understanding of the role of ion channels within the CCS will lead to the development of new treatments for cardiac diseases such as sick sinus syndrome, AV block, and tachycardia which origin is in the CCS.

L., Coetzee, W. A., Tristani-Firouzi, M., Bamshad, M. J., Christoffels, V. M., and Moon, A. M. (2011). Lethal arrhythmias in Tbx3deficient mice reveal extreme dosage sensitivity of cardiac conduction system function and homeostasis. Proc. Natl. Acad. Sci. U.S.A. 109, E154-E163.

Gourdie, R. G., Harris, B. S., Bond, J., Justus, C., Hewett, K. W., O’Brien, T. X., Thompson, R. P., and Sedmera, D. (2003). Development of the cardiac pacemaking and conduction system. Birth Defects Res. C Embryo Today 69, 46-57.

Haïssaguerre, M., Extramiana, F., Hocini, M., Cauchemez, B., Jaïs, P., Cabrera, J. A., Farré, J., Leenhardt, A., Sanders, P., Scavée, C., Hsu, L. F., Weerasooriya, R., Shah, D. C., Frank, R., Maury, P., Delay, M., Garrigue, S., and Clémenty, J. (2003). Mapping and ablation of ventricular fibrillation associated with long-QT and Brugada syndromes. Circulation 108, 925-928.

Hall, C. E., Hurtado, R., Hewett, K. W., Shulimovich, M., Poma, C. P., Reckova, M., Justus, C., Pennisi, D. J., Tobita, K., Sedmera, D., Gourdie, R. G., and Mikawa, T. (2004). Hemodynamic-dependent patterning of endothelin converting enzyme 1 expression and differentiation of impulse-conducting Purkinje fibers in the embryonic heart. Development 131, 581-592.

Hao, X., Zhang, Y., Zhang, X., Nirmalan, M., Davies, L., Konstantinou, D., Yin, F., Dobrzynski, H., Wang, X., Grace, A., Zhang, H., Boyett, M., Huang, C. L., and Lei, M. (2011). TGF- $\beta 1$-mediated fibrosis and ion channel remodeling are key mechanisms in producing the sinus node dysfunction associated with SCN5A deficiency and aging. Circ. Arrhythm. Electrophysiol. 4, 397-406.

Hatcher, C. J., and Basson, C. T. (2009). Specification of the cardiac conduction system by transcription factors. Circ. Res. 105, 620-630.

Herfst, L. J., Rook, M. B., and Jongsma, H. J. (2004). Trafficking and functional expression of cardiac Na+ channels. J. Mol. Cell. Cardiol. 36, 185-193.

Herrmann, S., Stieber, J., Stöckl, G., Hofmann, F., and Ludwig, A. (2007) HCN4 provides a "depolarization reserve" and is not required for heart rate acceleration in mice. EMBO J. 26, 4423-4432.

Hoesl, E., Stieber, J., Herrmann, S., Feil, S., Tybl, E., Hofmann, F., Feil, R., and Ludwig, A. (2008). Tamoxifen-inducible gene deletion in the cardiac conduction system. J. Mol. Cell. Cardiol. 45, 62-69.

Hoogaars, W. M., Engel, A., Brons, J. F., Verkerk, A. O., de Lange, F. J., Wong, L. Y., Bakker, M. L., Clout, D. E., Wakker, V., Barnett, P., Ravesloot, J. H., Moorman, A. F., Verheijck, E. E., and Christoffels, V. M. (2007). Tbx3 controls the sinoatrial node gene program and imposes pacemaker function on the atria. Genes Dev. 21, 1098-1112.

Hoogaars, W. M., Tessari, A., Moorman, A. F., de Boer, P. A., Hagoort, J., Soufan, A. T., Campione, M., and Christoffels, V. M. (2004). The transcriptional repressor $\mathrm{Tbx} 3$ delineates the developing central conduction system of the heart. Cardiovasc. Res. 62, 489-499. 
Kang, M., Giovannone, S. F., Liu, N., Liu, F. J., Zhang, J., Priori, S. G., and Fishman, G. I. (2010). Purkinje cells from RyR2 mutant mice are highly arrhythmogenic but responsive to targeted therapy. Circ. Res. 107, 512-519.

Kauferstein, S., Kiehne, N., Neumann, T., Pitschner, H., and Bratzke, H. (2009). Cardiac gene defects can cause sudden cardiac death in young people. Dtsch. Arztebl. Int. 106, 41-47.

Krous, H. F., Beckwith, J. B., Byard, R. W., Rognum, T. O., Bajanowski, T., Corey, T., Cutz, E., Hanzlick, R., Keens, T. G., and Mitchell, E. A. (2004). Sudden infant death syndrome and unclassified sudden infant deaths: a definitional and diagnostic approach. Pediatrics 114, 234-238.

Martin, C. A., Guzadhur, L., Grace, A. A., Lei, M., and Huang, C. L. (2011). Mapping of reentrant spontaneous polymorphic ventricular tachycardia in a Scn5a \pm mouse model. Am. J. Physiol. Heart Circ. Physiol. 300, H1853-H1862.
Mathur, N., Sood, S., Wang, S., van Oort, R. J., Sarma, S., Li, N., Skapura, D. G., Bayle, J. H., Valderrábano, M., and Wehrens, X. H. (2009). Sudden infant death syndrome in mice with an inherited mutation in RyR2. Circ. Arrhythm. Electrophysiol. 2, 677-685.

Moosmang, S., Stieber, J., Zong, X., Biel, M., Hofmann, F., and Ludwig, A. (2001). Cellular expression and functional characterization of four hyperpolarization-activated pacemaker channels in cardiac and neuronal tissues. Eur. J. Biochem. 268, 1646-1652.

Muir, A., Smith, G. L., Burton, F. L., Rankin, A. C., and Cobbe, S. M. (2005). Increased A-H interval in isolated $\mathrm{AV}$ node from rabbits with LV dysfunction due to myocardial infarction. J. Mol. Cell. Cardiol. 39, 197.

Nogami, A. (2001). Purkinje-related arrhythmias part II: polymorphic ventricular tachycardia and ventricular fibrillation. Pacing Clin. Electrophysiol. 34, 1034-1049.

Pennisi, D. J., Rentschler, S., Gourdie, R. G., Fishman, G. I., and
Mikawa, T. (2002). Induction and patterning of the cardiac conduction system. Int. J. Dev. Biol. 46, 765-775.

Priori, S. G., Napolitano, C., Tiso, N., Memmi, M., Vignati, G., Bloise, R., Sorrentino, V., and Danieli, G. A. (2001). Mutations in the cardiac ryanodine receptor gene (hRyR2) underlie catecholaminergic polymorphic ventricular tachycardia. Circulation 103, 196-192.

Stieber, J., Herrmann, S., Feil, S., Loster, J., Feil, R., Biel, M., Hofmann, F., and Ludwig, A. (2003). The hyperpolarization-activated channel HCN4 is required for the generation of pacemaker action potentials in the embryonic heart. Proc. Natl. Acad. Sci. U.S.A. 100, 15235-15240.

Thomas, G., Killeen, M. J., Gurung, I. S., Hakim, P., Balasubramaniam, R., Goddard, C. A., Grace, A. A., and Huang, C. L. (2007). Mechanisms of ventricular arrhythmogenesis in mice following targeted disruption of KCNE1 modelling
long-QT syndrome 5. J. Physiol. 578, 99-114.

Conflict of Interest Statement: The authors declare that the research was conducted in the absence of any commercial or financial relationships that could be construed as a potential conflict of interest.

Received: 14 March 2012; accepted: 29 May 2012; published online: 14 June 2012.

Citation: Aránega A, De La Rosa AJ and Franco D (2012) Cardiac conduction system anomalies and sudden cardiac death: insights from murine models. Front. Physio. 3:211. doi: 10.3389/fphys.2012.00211

This article was submitted to Frontiers in Cardiac Electrophysiology, a specialty of Frontiers in Physiology.

Copyright (c) 2012 Aránega, De La Rosa and Franco. This is an open-access article distributed under the terms of the Creative Commons Attribution Non Commercial License, which permits noncommercial use, distribution, and reproduction in other forums, provided the original authors and source are credited. 